\title{
Differential Synovial CGRP/RAMP1 Expression in Men and Women With Knee Osteoarthritis
}

Kentaro Uchida ${ }^{1}$, Shotaro Takano ${ }^{2}$, Ken Takata ${ }^{2}$, Manabu Mukai ${ }^{2}$, Tomohisa Koyama ${ }^{2}$, Yoshihisa Ohashi ${ }^{2}$, Hiroki Saito ${ }^{2}$, Masashi Takaso ${ }^{3}$, Masayuki Miyagi ${ }^{3}$, Gen Inoue ${ }^{4}$

1. Department of Orthopaedic Surgery, Kitasato University, School of Medicine, Sagamihara, JPN 2. Department of Orthopaedic Surgery, Kitasato University School of Medicine, Sagamihara, JPN 3. Department of Orthopedic Surgery, Kitasato University School of Medicine, Sagamihara, JPN 4. Orthopaedic Surgery, Kitasato University, Sagamihara, JPN

Corresponding author: Kentaro Uchida, kuchida@med.kitasato-u.ac.jp

\section{Abstract \\ Background}

Female patients with osteoarthritis report more severe knee pain compared to men. However, the mechanism underlying sex differences in pain remains unclear. We previously found that calcitonin generelated peptide (CGRP) was expressed in synovial tissue and that this localization may play a role in pain associated with knee osteoarthritis (KOA). Several animal studies have shown that the expression of CGRP and its receptor (receptor activity modifying protein 1, RAMP1) differs by sex. Here, we investigated synovial CGRP and RAMP1 expression in male and female patients with KOA.

\section{Methods}

Synovial tissue (ST) was harvested from male and female subjects ( $\mathrm{n}=30$ each) with radiographically confirmed unilateral Kellgren/Lawrence grade 3-4 KOA during total knee arthroplasty. Patients' subjective pain severity was scored on a 0 to $10 \mathrm{~cm}$ visual analog scale (VAS). We compared the expression of CGRP and RAMP1 in ST from men and women and examined the correlation between mRNA levels of CGRP and RAMP1 and pain severity.

\section{Results}

Synovial expression of CGRP and RAMP1 was significantly elevated in women compared to men (CGRP, $\mathrm{P}=0.017$; RAMP1, $\mathrm{P}=0.028$ ). While CGRP expression was positively correlated with pain severity in females $(\rho=0.443, P=0.014)$, no correlation was observed in men $(\rho=-0.021, P=0.913)$. RAMP1 expression was not correlated with pain severity in either men or women (male, $\rho=-0.114, P=0.939$; female, $\rho=-0.047, P=0.807$ ).

\section{Conclusion}

CGRP and RAMP1 expression levels differ between men and women. Differential CGRP levels may suggest the presence of different pain mechanisms in men and women with KOA.

Review began 05/19/2021 Review ended 05/25/2021 Published 06/06/2021

\section{() Copyright 2021}

Uchida et al. This is an open access article distributed under the terms of the Creative Commons Attribution License CC-BY 4.0., which permits unrestricted use, distribution, and reproduction in any medium, provided the original author and source are credited.
Categories: Orthopedics

Keywords: calcitonin gene-related peptide, receptor activity modifying protein 1, osteoarthritis, pain, synovium

\section{Introduction}

Compared to men, women have an elevated risk of developing knee osteoarthritis (KOA) [1, 2]. Additionally, women with osteoarthritis (OA) reported more severe knee pain and reduced function [3, 4]. Several studies have examined sex differences in pain sensitivity in healthy subjects. A meta-analysis identified elevated pain sensitivity in women compared to men based on their pain threshold towards various noxious stimuli [5]. Further, a clinical study demonstrated that women report more severe knee pain compared to men with a similar grade of radiographic KOA [6]. However, the mechanism underlying the difference in pain sensitivity between men and women remains unclear.

Calcitonin gene-related peptide (CGRP), a neuropeptide of 37 amino acids, binds to the calcitonin receptorlike receptor (CLR) and receptor activity-modifying protein 1 (RAMP1) [7]. With vasodilatory effects, CGRP is an important factor in migraine pain formation, with a clinical trial showing that small molecule CGRP receptor antagonists have beneficial effects in the treatment of migraine pain [8]. Additionally, studies have suggested that there may be an important link between synovial CGRP levels and OA-related pain [9, 10]. Interestingly, levels of CGRP and its receptors have been shown to differ by sex in rats and humans [11-16]. We hypothesized that the expression levels of CGRP and its receptors in osteoarthritic joints differ between men and women.

Herein, we investigated the synovial expression of CGRP and RAMP1 in male and female patients with knee 


\section{Cureus}

OA.

\section{Materials And Methods}

\section{Ethics approval and patient consent to participate}

Ethics approval was obtained from our institutional review board (IRB) (Approval number: B13-

113). Informed consent to participate in this study was obtained from all patients the day prior to surgery, in accordance with the Declaration of Helsinki. Patients were excluded based on the presence of specific comorbid conditions, including the following: metastatic cancer; rheumatoid arthritis, fibromyalgia, or other systemic rheumatic diseases; history of gout in knee or hip (more on this can be found in Material and Methods section of this article).

\section{Samples}

We harvested synovial tissue (ST) from the suprapatellar pouch of the operated knee of 60 patients (30 men and 30 women) with KOA during total knee replacement surgery. ST samples were instantly frozen in liquid nitrogen and then stored at $-80^{\circ} \mathrm{C}$ until RNA extraction.

\section{Real-time PCR}

Procedures used for RNA extraction, cDNA synthesis, and quantitative RT-PCR (qRT-PCR) were the same as those reported previously $[9,10,17,18]$. Primers against CGRP, RAMP1, and GAPDH were synthesized based on previous studies (Table 1) [9]. We evaluated levels of CGRP and RAMP1 mRNA in ST by dividing by levels of GAPDH using the $\triangle \triangle C T$ method (delta-delta cycle threshold method). We compared the expression of CGRP and RAMP1 in ST between the men and women in the study and evaluated the relationship between expression levels of CGRP and RAMP1 and pain, as determined using a 1-10 $\mathrm{cm}$ visual analog scale (VAS). To evaluate the effect of OA severity on CGRP and RAMP1 expression, we stratified patients based on KOA severity and compared those with Kellgren/Lawrence (KL) grade 3 to those with KL 4.

\begin{tabular}{|c|c|c|c|}
\hline Gene & Direction & Primer Sequence $(5 \$-3 \phi)$ & Product Size (bp) \\
\hline \multirow{2}{*}{ CGRP } & $\mathrm{F}$ & IIGCCCAGAAGAGAGCCIGIG & \multirow{2}{*}{91} \\
\hline & $\mathbf{R}$ & TTGTTCTTCACCACACССССТG & \\
\hline \multirow{2}{*}{ RAMP1 } & $\mathrm{F}$ & GGCCTCTGGCTGCTCCTG & \multirow{2}{*}{172} \\
\hline & $\mathbf{R}$ & GCTCCCTGTAGCTCCTGATG & \\
\hline \multirow{2}{*}{ GAPDH } & $\mathrm{F}$ & TGTTGCCATCAATGACCCCTT & \multirow{2}{*}{202} \\
\hline & $\mathbf{R}$ & CTCCACGACGTACTCAGCG & \\
\hline
\end{tabular}

TABLE 1: Sequences of the primers used in this study

\section{Statistical analysis}

All statistical analyses were conducted using SPSS 25.0. Categorical and continuous variables were compared using the chi-squared test and Mann-Whitney U test, respectively. The relationship between CGRP and RAMP1 and pain severity was evaluated using Spearman's correlation coefficient. Statistical significance was defined as $\mathrm{P}<0.05$.

\section{Results}

\section{Expression of CGRP and RAMP1 in ST from male and female KOA patients}

We studied CGRP and RAMP1 expression in ST from male and female KOA patients. Age, bone mass index (BMI), K/L grade 3/4 ratio, and VAS score were comparable between sexes (Table 2). In contrast, CGRP and RAMP1 expression were significantly higher in women than in men (CGRP, $\mathrm{P}=0.017$; RAMP1, $\mathrm{P}=0.028$; Figure 1). 


\section{Cureus}

\begin{tabular}{|c|c|c|c|}
\hline & Male & Female & P-value \\
\hline Age (years) & $72.9 \pm 10.5$ & $74.4 \pm 6.7$ & 0.824 \\
\hline $\mathrm{BMI}\left(\mathrm{kg} / \mathrm{m}^{2}\right)$ & $27.1 \pm 3.2$ & $26.6 \pm 4.3$ & 0.258 \\
\hline KL grade $(3,4) n$ & 11,19 & 13,17 & 0.792 \\
\hline $\operatorname{VAS}(\mathrm{cm})$ & $6.4 \pm 2.5$ & $7.3 \pm 2.3$ & 0.149 \\
\hline
\end{tabular}

TABLE 2: Patients' demographics

BMI, bone mass index; K/L, Kellgren/Lawrence; VAS, visual analog scale
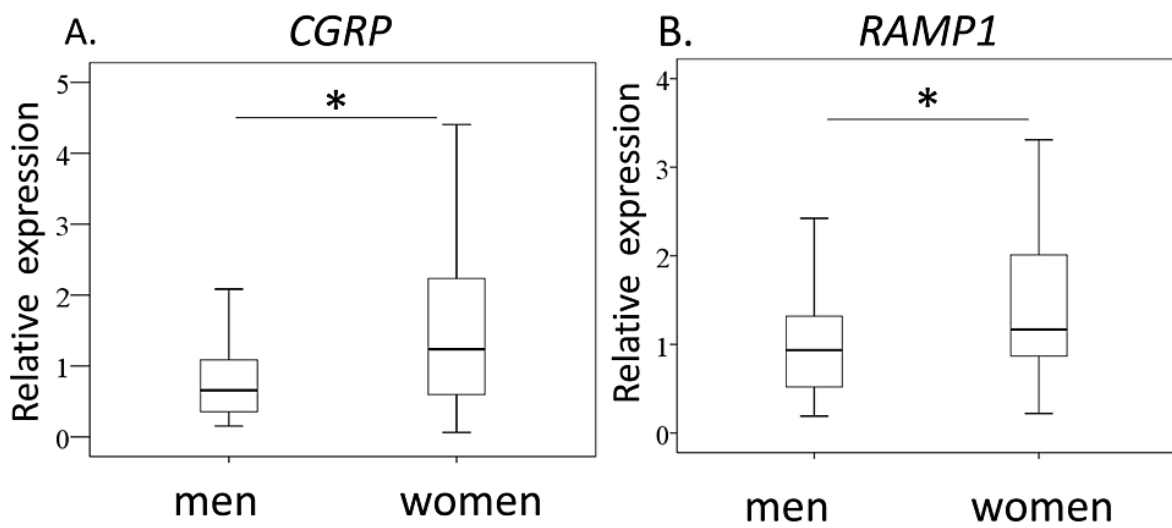

FIGURE 1: Synovial CGRP and RAMP1 expression men and women with knee osteoarthritis

Quantitative RT-PCR analysis for CGRP (A) and RAMP1(B). * $\mathrm{P}<0.05$

Relationship between CGRP and RAMP1 expression and pain severity in men and women

While CGRP expression was positively correlated with pain severity in women ( $\rho=0.443, \mathrm{P}=0.014)$, no correlation was observed in men $(\rho=-0.021, P=0.913)$. Further, RAMP1 expression was not correlated with pain severity in men or women (male, $\rho=-0.114, \mathrm{P}=0.939$; female, $\rho=-0.047, \mathrm{P}=0.807$; Figure 2). 


\section{Cureus}
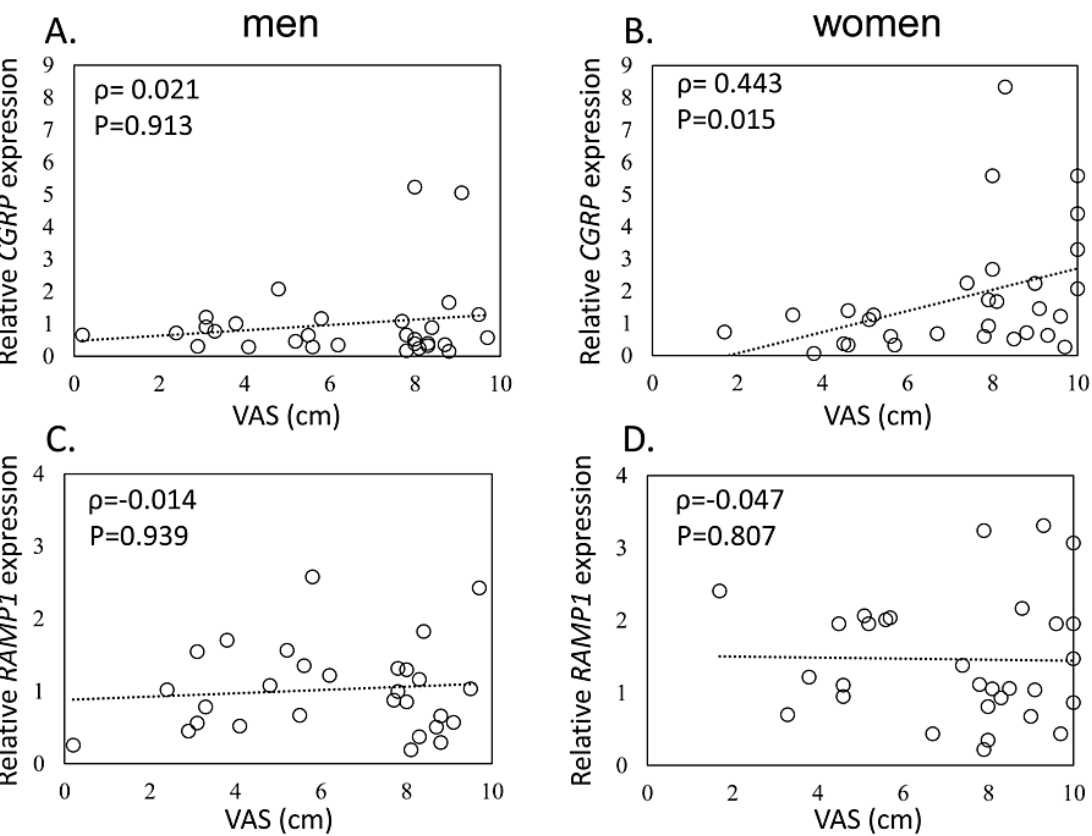

FIGURE 2: Relationship between CGRP and RAMP1 expression and pain severity in men and women

Relationship between visual analog scale (VAS) and CGRP in men (A) and women (B), and RAMP1 in men (C) and women (D)

\section{Effect of OA severity on CGRP and RAMP1 expression}

Age, BMI, and VAS score were comparable between patients with KL3 and KL4 for both men and women (Table 3). Likewise, there was no significant difference in CGRP or RAMP1 expression between patients with KL3 and KL4 for both men and women (Figures 3A-D).

\begin{tabular}{|c|c|c|c|c|c|c|}
\hline & \multicolumn{2}{|l|}{ Male } & \multirow[b]{2}{*}{ P-value } & \multicolumn{2}{|l|}{ Female } & \multirow[b]{2}{*}{ P-value } \\
\hline & KL3 (n=11) & KL4 (n=19) & & KL3 $(n=13)$ & KL4 (n=17) & \\
\hline Age (years) & $68.9 \pm 11.4$ & $75.6 \pm 9.1$ & 0.085 & $72.3 \pm 6.7$ & $76.1 \pm 6.5$ & 0.333 \\
\hline BMI $\left(\mathrm{kg} / \mathrm{m}^{2}\right)$ & $28.1 \pm 2.6$ & $26.6 \pm 3.5$ & 0.149 & $27.1 \pm 4.1$ & $26.3 \pm 4.6$ & 0.338 \\
\hline VAS (cm) & $5.8 \pm 2.9$ & $6.7 \pm 2.3$ & 0.438 & $7.9 \pm 2.1$ & $6.8 \pm 2.4$ & 0.180 \\
\hline
\end{tabular}

TABLE 3: Comparison of radiographic osteoarthritis severity and demographics between male and female patients

BMI, bone mass index; VAS, visual analog scale. 
A.

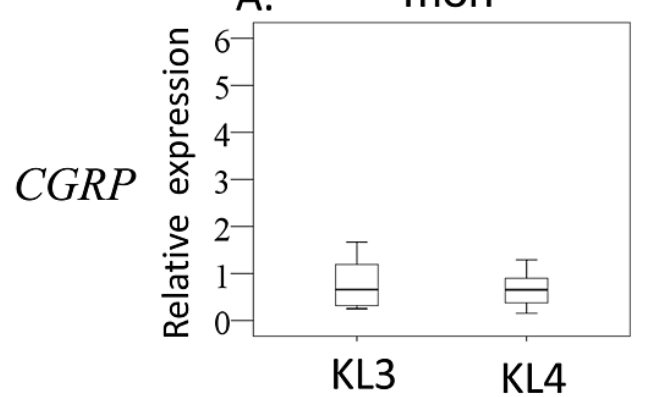

C.

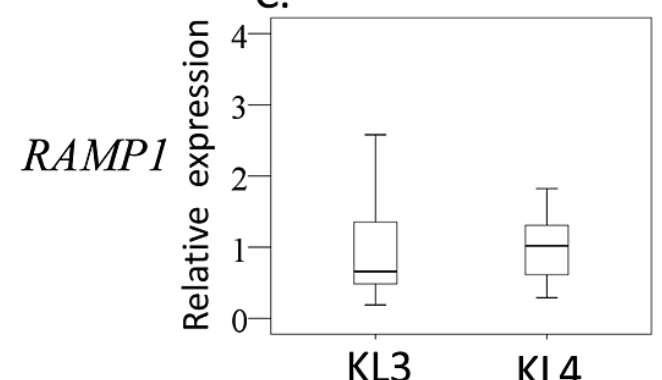

B.

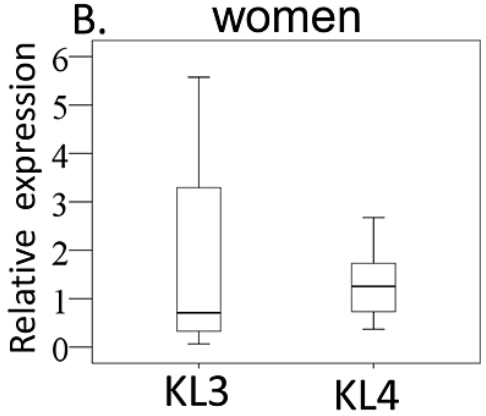

D.

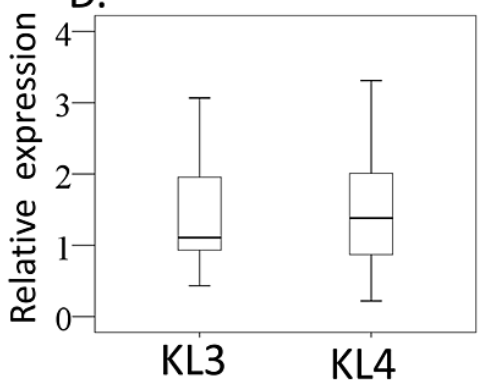

FIGURE 3: Effect of OA severity on CGRP and RAMP1 expression

Effect of OA severity on CGRP expression in men (A) and women (B) and RAMP1 expression in men (C) and women (D).

\section{Discussion}

Previous studies have reported that the expression of CGRP and RAMP1 differs by sex and is affected by sex hormone levels in serum and several tissues [11-13, 16, 19]. Women have higher plasma CGRP levels than men [14]. Further, an estrogen deficiency has been shown to increase CGRP levels within the lumbar dorsal root ganglion (DRG), medial preoptic nucleus of the hypothalamus, as well as in the midbrain periaqueductal gray in rats $[12,16]$. Meanwhile, estrogen treatment in rats leads to reduced CGRP expression in the DRG and trigeminal nucleus caudalis [13]. In contrast, a previous study showed that ovariectomy significantly raises CGRP levels in the trigeminal ganglia relative to the control rats and that estrogen replacement suppresses this increase [11]. Moreover, treatment of ovariectomized rats with estradiol-17 $\beta$ lowered RAMP1 mRNA expression in uterine tissues [19]. In our study, female KOA patients had higher synovial CGRP and RAMP1 expression levels than male patients. As all the female KOA patients were postmenopausal, we predict that the increase in synovial CGRP and RAMP1 expression may have been caused by estrogen deficiency.

In our previous studies, we found that CGRP was expressed in ST and that this expression was correlated with pain in KOA patients comprising both men and women $[9,10]$. In the present study, we found that synovial CGRP expression level was correlated with the VAS pain score in women but not men. A previous study suggested that sensitivity toward CGRP differed between male and female rats, with mesenteric arteries isolated from females showing heightened vasorelaxation sensitivity to CGRP [20]. As RAMP1 expression was also higher in women than men in our study, sensitivity to CGRP may partly explain the correlation between CGRP expression and pain in women but not men.

Two limitations of the present study warrant mention. First, the present study lacked healthy control data. Second, the sample size was small $(\mathrm{n}=30)$.

\section{Conclusions}

Female KOA patients had higher CGRP and RAMP1 expression in ST than men, and CGRP expression level correlated with pain severity. Sex differences in the CGRP pathway may provide valuable insight for the development of gender-specific treatments.

\section{Additional Information}

\section{Disclosures}

Human subjects: Consent was obtained or waived by all participants in this study. Kitasato University 
Institutional Review Board issued approval B13-113. Ethics approval was obtained from the Kitasato University Institutional Review Board (IRB) (Approval number: B13-113). Animal subjects: All authors have confirmed that this study did not involve animal subjects or tissue. Conflicts of interest: In compliance with the ICMJE uniform disclosure form, all authors declare the following: Payment/services info: All authors have declared that no financial support was received from any organization for the submitted work. Financial relationships: All authors have declared that they have no financial relationships at present or within the previous three years with any organizations that might have an interest in the submitted work. Other relationships: All authors have declared that there are no other relationships or activities that could appear to have influenced the submitted work.

\section{Acknowledgements}

This investigation was supported in part by a Grant-in-Aid for Young Scientists Grant No. 20K18073, Grantin-Aid for Young Scientists (Start-up) Grant No. 19K24037, Grant-in-Aid for Scientific Research (C) Grant No. 18K09119, Kitasato University Research Grant for Young Researchers, and research grants from the Parents' Association of Kitasato University School of Medicine.

\section{References}

1. Felson DT, Zhang Y, Hannan MT, Naimark A, Weissman B, Aliabadi P, Levy D: Risk factors for incident radiographic knee osteoarthritis in the elderly: the Framingham Study. Arthritis Rheum. 1997, 40:728-33. 10.1002/art.1780400420

2. Oliveria SA, Felson DT, Reed JI, Cirillo PA, Walker AM: Incidence of symptomatic hand, hip, and knee osteoarthritis among patients in a health maintenance organization. Arthritis Rheum. 1995, 38:1134-41. 10.1002/art.1780380817

3. Ebrahimpour PB, Do HT, Bornstein LJ, Westrich GH: Relationship between demographic variables and preoperative pain and disability in 5945 total joint arthroplasties at a single institution. J Arthroplasty. 2011, 26:133-137.e1. 10.1016/j.arth.2011.04.011

4. Hawker GA, Wright JG, Coyte PC, Williams JI, Harvey B, Glazier R, Badley EM: Differences between men and women in the rate of use of hip and knee arthroplasty. N Engl J Med. 2000, 342:1016-22. 10.1056/NEJM200004063421405

5. Riley JL, 3rd, Robinson ME, Wise EA, Myers CD, Fillingim RB: Sex differences in the perception of noxious experimental stimuli: a meta-analysis. Pain. 1998:181-7. 10.1016/s0304-3959(97)00199-1

6. Glass N, Segal NA, Sluka KA, et al.: Examining sex differences in knee pain: the multicenter osteoarthritis study. Osteoarthritis Cartilage. 2014, 22:1100-6. 10.1016/j.joca.2014.06.030

7. Walker CS, Conner AC, Poyner DR, Hay DL: Regulation of signal transduction by calcitonin gene-related peptide receptors. Trends Pharmacol Sci. 2010, 31:476-83. 10.1016/j.tips.2010.06.006

8. Doods H, Arndt K, Rudolf K, Just S: CGRP antagonists: unravelling the role of CGRP in migraine . Trends Pharmacol Sci. 2007, 28:580-7. 10.1016/j.tips.2007.10.005

9. Minatani A, Uchida K, Inoue G, et al.: Activation of calcitonin gene-related peptide signaling through the prostaglandin E2-EP1/EP2/EP4 receptor pathway in synovium of knee osteoarthritis patients. J Orthop Surg Res. 2016, 11:117. 10.1186/s13018-016-0460-4

10. Takano S, Uchida K, Inoue G, et al.: Increase and regulation of synovial calcitonin gene-related peptide expression in patients with painful knee osteoarthritis. J Pain Res. 2017, 10:1099-104. 10.2147/JPR.S135939

11. Aggarwal M, Puri V, Puri S: Effects of estrogen on the serotonergic system and calcitonin gene-related peptide in trigeminal ganglia of rats. Ann Neurosci. 2012, 19:151-7. 10.5214/ans.0972.7531.190403

12. Herbison AE, Spratt DP: Sexually dimorphic expression of calcitonin gene-related peptide (CGRP) mRNA in rat medial preoptic nucleus. Brain Res Mol Brain Res. 1995, 34:143-8. 10.1016/0169-328x(95)00144-h

13. Pardutz A, Multon S, Malgrange B, Parducz A, Vecsei L, Schoenen J: Effect of systemic nitroglycerin on CGRP and 5-HT afferents to rat caudal spinal trigeminal nucleus and its modulation by estrogen. Eur J Neurosci. 2002, 15:1803-9. 10.1046/j.1460-9568.2002.02031.x

14. Valdemarsson S, Edvinsson L, Hedner P, Ekman R: Hormonal influence on calcitonin gene-related peptide in man: effects of sex difference and contraceptive pills. Scand J Clin Lab Invest. 1990, 50:385-8. 10.3109/00365519009091595

15. Wang D, Zhao J, Wang J, Li J, Yu S, Guo X: Deficiency of female sex hormones augments PGE2 and CGRP levels within midbrain periaqueductal gray. J Neurol Sci. 2014, 346:107-11. 10.1016/j.jns.2014.08.002

16. Yang Y, Ozawa H, Lu H, et al.: Immunocytochemical analysis of sex differences in calcitonin gene-related peptide in the rat dorsal root ganglion, with special reference to estrogen and its receptor. Brain Res. 1998, 791:35-42. 10.1016/s0006-8993(98)00021-3

17. Takata K, Uchida K, Mukai M, et al.: Increase in tryptase and its role in the synovial membrane of overweight and obese patients with osteoarthritis of the knee. Diabetes Metab Syndr Obes. 2020, 13:1491-7. 10.2147/DMSO.S253147

18. Uchida K, Takano S, Inoue G, et al.: Increase in mast cell marker expression in the synovium of obese patients with osteoarthritis of the knee. Diabetes Metab Syndr Obes. 2019, 12:377-82. 10.2147/DMSO.S201523

19. Thota C, Gangula PR, Dong YL, Yallampalli C: Changes in the expression of calcitonin receptor-like receptor, receptor activity-modifying protein (RAMP) 1, RAMP2, and RAMP3 in rat uterus during pregnancy, labor, and by steroid hormone treatments. Biol Reprod. 2003, 69:1432-7. 10.1095/biolreprod.103.015628

20. Gangula PR, Lanlua P, Bukoski RD, Wimalawansa SJ, Yallampalli C: Mesenteric arterial relaxation to calcitonin gene-related peptide is increased during pregnancy and by sex steroid hormones. Biol Reprod. 2004, 71:1739-45. 10.1095/biolreprod.104.031369 